\title{
Application of human bone marrow-derived mesenchymal stem cells in the treatment of radiation-induced gastrointestinal syndrome
}

\author{
YANG Chao $^{1}$, DAI WeiMin ${ }^{2}$, CHEN HaiXu ${ }^{3} \&$ WU BenYan ${ }^{1 *}$ \\ ${ }^{1}$ Gastrointestinal Department of Southern Building, General Hospital of Chinese PLA, Beijing 100853, China; \\ ${ }^{2}$ Department of Thoracic Surgery, General Hospital of Chinese PLA, Beijing 100853, China; \\ ${ }^{3}$ Institute of Gerontology and Geriatrics, General Hospital of Chinese PLA, Beijing 100853, China
}

Received March 26, 2013; accepted October 22, 2013; published online September 5, 2014

\begin{abstract}
Nuclear accidents and terrorism present a serious threat for mass casualty. Accidental or intended radiation exposure leads to radiation-induced gastrointestinal (GI) syndrome. However, currently there are no approved medical countermeasures for GI syndrome. Thus, developing novel treatments for GI syndrome is urgent. Mesenchymal stem cells (MSCs) derived from bone marrow are a subset of multipotent adult somatic stem cells that have the ability to undergo self-renewal, proliferation and pluripotent differentiation. MSCs have advantages over other stem cells; they can be easily isolated from patients or donors, readily expanded ex vivo, and they possess reparative and immunomodulatory properties. Moreover, MSCs have been shown to be powerful tools in gene therapy and can be effectively transduced with vectors containing therapeutic genes. Therefore, the therapeutic potential of MSCs has been brought into the spotlight for the clinical treatment of GI syndrome. In this review, we discuss the possible role of MSCs in radiation-induced GI syndrome.
\end{abstract}

mesenchymal stem cells, radiation-induced gastrointestinal syndrome, treatment

Citation: Yang C, Dai WM, Chen HX, Wu BY. Application of human bone marrow-derived mesenchymal stem cells in the treatment of radiation-induced gastrointestinal syndrome. Sci China Life Sci, 2014, 57: 1177-1182, doi: 10.1007/s11427-014-4721-3

Accidental or intended radiation exposure in a mass casualty setting presents a serious and ongoing threat. Exposure to ionizing radiation not only causes apoptosis of intestinal crypt cells and functional changes, but also results in a complex interplay of pathophysiological processes. These deleterious effects, including inflammatory responses, diarrhea, hematochezia, and malabsorption, are seen clinically as radiation-induced gastrointestinal (GI) syndrome [1-3]. The acute phase symptoms of GI syndrome may persist for a short time, but long-term complications can represent significant clinical conditions with high morbidity. However, the treatments usually applied to manage GI syndrome are only symptomatic [4]. Therefore, developing novel and effective therapeutics for radiation-induced GI syndrome is necessary and important. Mesenchymal stem cells (MSCs) are non-hematopoietic stem cells, first isolated from bone marrow, with subsequent isolation from other adult tissues. They exhibit the capacity for self-renewal, proliferation and differentiation into various cells under appropriate stimuli. MSCs are capable of self-replication and multi-lineage differentiation [5,6]. Stem cell-based approaches using MSCs are promising for the development of GI syndrome therapy. The aim of this review article is to illustrate the potential of MSCs in the treatment of GI syndrome.

*Corresponding author (email: benyanwu@vip.sina.com) 


\section{Overview of GI syndrome}

More than $60 \%$ of patients with abdominal or pelvic tumors might receive radiotherapy. However, GI syndrome is a major limiting factor in abdominal and pelvic radiotherapy. GI syndrome occurs in more than $10 \%-20 \%$ of patients who have undergone abdominopelvic radiotherapy. GI epithelial cells are one of the most radiosensitive cell types, especially intestinal epithelial stem cells. Ionizing irradiation induces cellular, tissue and organ toxicity. The generation of reactive oxygen species (ROS) not only contributes to apoptosis and functional changes of intestinal crypt cells, but also results in a complex interplay of pathophysiological processes [7]. Currently, there are no approved medical countermeasures to alleviate GI syndrome. Several studies have shown that cytokines such as interleukin 11 (IL-11), granulocyte colony-stimulating factor (G-CSF) and basic fibroblast growth factor (b-FGF) could inhibit apoptosis of intestinal epithelial cells and impair radiation-induced intestinal damage $[8,9]$. However, cytokine treatment is not effective against higher doses of radiation-induced tissue injury. Therefore, interventions that reduce intestinal radiation injury are urgently needed.

\section{The pathogenesis of GI syndrome}

The pathogenesis of radiation-induced GI syndrome remains underdeveloped [10]. The GI tract is particularly radiosensitive. Exposure to ionizing radiation kills crypt stem cells or villus endothelial cells. Destructive changes of the GI epithelial cells cause breakdown of the mucosal barrier, resulting in severe secretory diarrhea, dehydration and fluid-electrolyte imbalance, leading to prominent features including vascular damage, smooth muscle degeneration and intestinal wall fibrosis $[11,12]$. Ionizing radiation initiates inflammation by the production of ROS and induces double-strand breaks in DNA, mucosal breakdown, or necrosis [13]. Several molecular pathways have been implicated in regulating radiation-induced apoptosis in crypt cells, including those of the tumor suppressors, p53 and Ataxia telangiectasia-mutated (ATM) P53, which have been suggested to play a key role in determining the fate of cells that have potentially received DNA damage. P53 transactivates p21, which results in delayed progression during S-phase of the cell cycle. This is considered to be one of the main mechanisms of G1-phase arrest in crypt precursor cells [14]. Knocking out pro-apoptotic genes, Bax or Bak, results in apoptosis of vascular endothelial cells of the GI tract, following intestinal radiation injury in mice [15]. Others believed that endothelial apoptosis, independent of p53, is involved in the pathogenesis of GI syndrome, and suggest that radiation-targeted cells switch from endothelial cells to epithelial cells at higher doses of radiation [16]. Acid sphingomyelinase (ASMase)-mediated ceramide production is suggested to be responsible for apoptosis, in both endothelial cells and epithelial cells. b-FGF promotes stem cell renewal, progenitor cell differentiation and epithelial cell proliferation. Systemic administration of b-FGF is capable of suppressing ASMase in endothelial cells, which in turn was found to protect the murine small intestine from radiation-induced damage [17,18]. Zhang et al. [17] have shown that survival of crypt epithelial cells is significantly increased and apoptosis of endothelial cells pharmacologically inhibited in mice by intravenous injection of b-FGF prior to irradiation. Endothelial dysfunction likely plays a key role in both early and delayed radiation responses in the intestine. Radiation induces alterations in expression of the apoptosis-related proteins, C-fos, Jas/Fasl and P53, which eventually results in endogenous damage and delayed endogenous intestinal epithelial apoptosis. P53-activation also leads to cell cycle arrest and DNA double-strand break repair, or apoptosis. Caspase-3 may play an important role in the physiological process of apoptosis, which involves activation of caspases, constitutively expressed in most cells of the crypt and villus [19]. The BH3-only members of the Bcl-2 protein family and p53 up-regulate modulator of apoptosis (PUMA) are expressed at the early stage of apoptosis. PUMA has been shown by multiple research groups to play an essential role in both P53-dependent and P53-independent apoptosis of intestinal stem cells (ISCs) [20]. P53 levels increase within minutes of DNA damage, as well as PUMA, NOXA and other members of the bck-2 family [21]. Upon transcriptional induction in response to radiation-induced DNA damage, PUMA functions through other Bcl-2 family members, including Bax, Bcl-2 and $\mathrm{Bcl}-\mathrm{xL}$, to induce mitochondrial dysfunction and caspase activation. Qiu and colleagues [22] have shown that PUMA-deficient mice exhibit blocked crypt apoptosis following radiation, and that suppressing PUMA leads to radio-protection and prolonged survival in ISCs. P53-mediated growth arrest following DNA damage occurs in ISCs. It therefore appears that regulation of P53 activity in ISCs has emerged as a key concept in the injury response of these progenitor cells. The nuclear factor $\kappa \mathrm{B}(\mathrm{NF}-\kappa \mathrm{B})$ signaling pathway may also be involved in GI syndrome injury [23]. As well as deepening understanding of the pathogenesis mechanisms of GI syndrome, it is possible to open up new perspectives for the treatment of GI syndrome.

\section{Biological characteristics of MSCs}

Bone marrow-derived MSCs are a heterogeneous subpopulation of non-hematopoietic stem cells. There is no single surface marker specific for MSCs. Generally, MSCs derived from standard cultures have been defined by their homogeneous expression of surface markers, such as CD29, CD44, CD90, CD73 and CD105, and lack in expression of CD34, 
CD45, CD14, B7-1, B7-2 and HLA-DR [24]. MSCs have been shown to be able to differentiate into various cell lineages, including osteoblasts, adipocytes, chondrocytes, myoblasts and fibroblasts [25,26], as well as cells of the GI epithelial, liver, skin, lung, pancreas and nerve lineage [27-29].

In addition to the aforementioned characteristics, MSCs have advantages over other stem cells, in that they can be easily isolated from patients or donors and efficiently gene-engineered. In the past few years, the use of MSCs in gene therapy has gained momentum. Many reports have shown that MSCs were useful as vehicles for cell and gene therapy. The strategies for gene delivery into MSCs include using viral vectors, non-vectors and three-dimensional/ reverse transfection systems. The resulting expression of exogenous genes in transfected MSCs enhances tissue specificity. Overexpression of chemokine receptor 1 (CCR1), the receptor for CCL7, or chemokine receptor 2 (CXCR2), the receptor for both CXCL1 and CXCL2, enhances the migration, survival and engraftment of MSCs, and may provide a new therapeutic strategy for the injured myocardium [30]. MSCs can serve as cellular vehicles for gene delivery and are being developed to treat various diseases. Previous studies report that MSCs accelerate epithelial renewal and absorptive functions of the intestine in GI syndrome by reducing the level of proinflammatory cytokines, while secreting factors that support the regeneration of ISCs and their niche [30,31]. Thus, stem cell therapy using MSCs may be a very attractive option for treatment of GI syndrome.

\section{Therapeutic potential of MSCs in GI syn- drome}

MSCs are promising for the development of future therapies, based on their capacity to differentiate into multi-lineage cell types and also act as bioreactors of soluble factors to promote tissue regeneration at the injured sites. Several studies demonstrate that MSCs migrate to sites of local tissue damage or inflammation. For example, Kudo et al. [32] provide evidence that injection of MSCs into the wall of the irradiated (30 Gy) intestine of mice immediately improves survival rates and recovery of the intestinal injury. Sémont et al. [33] report that infusion of MSCs prolongs the lifespan of mice and improves the renewal capability of the small intestinal epithelium and small intestinal structure. Total body irradiation (TBI) has been shown to enhance MSC transplantation in the bone marrow and muscle, leading to further engraftment in the bone marrow, brain, heart, lung, liver and intestine [34,35]. Local irradiation in addition to TBI increases homing of injected cells to the injured tissues and to other tissues outside the local irradiation field [36]. Our group has previously shown that human MSCs delivered to NOD/SCID mice with GI syndrome via the intravenous route were distributed within the GI tract and proliferated in vivo [37].

However, it is important to note that the engraftment rate of MSCs in the intestinal mucosa is generally low, ranging from $0.17 \%-2.7 \%$ [38]. Thus, rarity of cell engraftment is the key problem that hampers the practical application of MSCs to GI syndrome, and more efforts should be focused on enhancing levels of engraftment. The stromal cellderived factor-1 (SDF-1)/CXC receptor 4 (CXCR4) axis plays an essential role in mobilization and engraftment of stem cells $[39,40]$. SDF-1 is a homeostatic chemokine. The increase in inflammatory chemokine concentration of SDF-1 at the site of inflammation is a crucial mediator in trafficking of MSCs to the site of tissue damage, such as after heart infarct, ischemia, irradiation and toxic liver damage [41]. It is well known that MSCs express the specific SDF-1 chemokine receptor, CXCR4, and migrate to injured sites by SDF-1/CXCR4 axis. Many groups have attempted to modify the functional properties of MSCs to increase their homing potential. Overexpression of CXCR4 with retroviral vector significantly enhanced the engraftment rate of MSCs into enteric mucosa, and the implanted MSCs effectively ameliorated radiation-induced intestinal injury [42]. Although the SDF-1/CXCR4 axis has been well characterized as a pathway for MSC homing, several other ligand-receptor interactions have also recently been shown to be involved in MSC homing [43]. Sasaki et al. [44] recently demonstrated that infused-MSCs significantly contribute to wound repair via MSC accumulation in the wound site. Many lines of evidence suggest that MSCs produce several essential growth factors, including hepatocyte growth factor (HGF), transforming growth factor (TGF) and granulocyte-macrophage colony-stimulating factor (GMCSF), as well as hematopoietic growth factors IL-6, IL-7, IL-8, IL-11, IL-12, IL-14, and stem cell factor (SCF) [41]. Lange et al. [45] showed long-term survival of mice after lethal irradiation, with fast hematopoietic recovery after exclusive transplantation of MSCs, without support of hematopoietic stem cells (HSCs). In comparison to HSCtransplanted animals, MSC-transplanted animals display enhanced regenerative features, characterized by decreased proinflammatory cytokines, enhanced extracellular matrix (ECM) formation and adhesion properties and boosted anti-inflammation, detoxification, cell cycle and anti-oxidative stress control [46].

Previous studies report that MSCs lack expression of MHC class II and most of the classical co-stimulatory molecules, such as CD80, CD86 and CD40 [47,48]. MSCs have been shown to regulate the activity in a range of effector cells involved in both innate and adaptive immunities. MSCs could inhibit up-regulation of CD1a, CD40, CD80, and CD86 during dendritic cell (DC) maturation. Different studies report that MSCs have an immunomodulatory effect and are able to suppress proliferation of T-lymphocytes in vitro [48-50]. MSCs can inhibit T-cell function, shift the 
T-helper lymphocyte balance and induce T-cell apoptosis [51]. Di Nicola et al. [52] further identified that MSCs mediate their suppressive effect by suppression of T-cell proliferation or through modulation of antigen-presenting cell phenotype and function. This immunosuppression has been shown to be mediated by cell-contact-dependent and -independent mechanisms, through the release of soluble factors. The interaction between MSCs and natural killer (NK) cells may contribute to the immunomodulatory effect of MSCs [53]. Crosstalk between MSCs and cells of the immune system leads to increased secretion of soluble immunomodulatory factors [54]. However, further research needs to be done to successfully identify the requirement of cell-to-cell contact.

The therapeutic efficacy of MSCs anti-inflammatory properties has been established in a number of preclinical models including graft versus host disease, inflammatory bowel disease, sepsis and allergic airway disease [53-55]. Furthermore, radio protective gene therapy as a therapeutic paradigm holds tremendous promise for patients who undergo radiotherapy with various types of malignancies. Accordingly, stem cell therapy using genetically modified MSCs may be an attractive and potential option for GI syndrome.

The generation of ROS is considered as the main cause of ionizing radiation-induced cellular, tissue and organ toxicity. It not only contributes to apoptosis and functional changes of intestinal crypt cells, but also results in a complex interplay of pathophysiological processes. These deleterious effects, including inflammatory responses, diarrhea, hematochezia and malabsorption, are seen clinically as acute small bowel reactions. To suppress the toxic effects of radiation, gene therapy approaches are being developed to deliver proteins that suppress ROS toxicity. Manganese superoxide dismutase (MnSOD) is the major antioxidant enzyme localized in the mitochondria, catalyzing the dismutation of superoxide into oxygen and hydrogen peroxide, and protecting mitochondria against ROS [56]. Intraesophageal administration of MnSOD-plasmid/liposome (MnSOD-PL) reduces esophageal epithelial damage and improves migration of restorative bone marrow progenitors to the esophageal squamous epithelium. Niu et al. [57] demonstrate the efficacy of MnSOD-PL in suppressing radiation-induced recombination in vivo. MnSOD has been shown to ameliorate radiation-induced injury through targeted localization to the mitochondrial membrane. Gene therapy using MnSOD-PL protects against radiationinduced tissue damage and suppresses radiation-induced inflammation, suggesting that expression of the MnSOD transgene reduces levels of ROS [58]. Administration of the MnSOD transgene in liposomes/plasmid or adenovirus reduces both the acute and chronic toxicity of irradiation in the mouse model $[59,60]$. Overexpression of the MnSOD gene, through the use of either adenoviral or retroviral vectors, has been shown to ameliorate radiation-induced inju- ries in the brain, lung, oral cavity mucosa and skin. More recently, our group has demonstrated that overexpression of human MnSOD in MSCs protects against tert-butyl hydroperoxide (t-BHP)-induced apoptosis in vitro [61]. Furthermore, we showed that administration of MnSOD-MSCs extends the life span of irradiated mice. MSC migrates to the small intestine, encourages the repair of radiationinduced intestinal structural damage, and inhibits radiation-induced apoptosis in a process involving the inhibition of cleaved caspase-3 activity [62]. These results indicate that gene therapy using MnSOD-PL protects against radiation-induced tissue damage and suppresses radiationinduced inflammation, suggesting that expression of the MnSOD transgene reduces levels of ROS.

All these findings suggest that MSCs have therapeutic potential in GI syndrome. Microenvironmental changes, induced by tissue injury, may play a key role in the homing and recruitment of circulating MSCs. MSCs have been shown to secrete massive amounts of molecules, including bioactive and ECM factors. Interestingly, the secretion of bioactive factors by MSCs is regulated in a manner closely associated with their growth and differentiation. These effects exhibit a common trophic mode of action: (i) inhibition of apoptosis and limitation of the local injury; (ii) inhibition of fibrosis or scarring at sites of damage; (iii) stimulation of mitosis of tissue-specific and endogenous stem cells.

Based on the literature discussed herein, the mechanism of action for MSCs in GI syndrome is proposed as follows. MSCs migrate to irradiation exposed sites, where they produce vital biomolecules and antioxidants by the paracrine system. The MSCs then transdifferentiate to promote reconstruction of the enteric microenvironment and modulate the enteric immunity, possibly to form intestinal functional cells.

\section{Prospects for future research}

MSCs are adult stem cells most commonly isolated from bone marrow that possess unique immunomodulatory and paracrine properties, which make them attractive for a wide range of diseases in regenerative medicine. Pioneer in vivo studies have mainly focused on their ability to engraft to the injured GI tract and promote structural and functional repair of damage tissues. The ability to efficiently transfer genes of interest into these cells would create a number of therapeutic opportunities. Together, these characteristics make MSCs an ideal candidate for treatment application in GI syndrome. Of the proposed mechanisms of action used by MSC, immunomodulation has been somewhat elucidated in vitro; however, data from preclinical transplant models regarding this are unclear. Furthermore, the optimal timing, dose and route of administration of MSCs remain to be elucidated. In summary, preclinical studies still need to be performed for the safe use of MSCs in future clinical applications. 
1 Gavazzi C, Bhoori S, Lovullo S, Cozzi G, Mariani L. Role of home parenteral nutrition in chronic radiation enteritis. Am J Gastroenterol, 2006, 101: 374-379

2 Vidal A, de la Cuerda C, Luis Escat J, Breton I, Camblor M, Garcia-Peris P. Chronic radiation enteritis after ovarian cancer: from home parenteral nutrition to oral diet. Clin Nutr, 2006, 25: 701-704

3 Frisby CL, Fraser RJ, Schirmer MB, Yeoh EK, Blackshaw LA. Roles of muscarinic receptor subtypes in small intestinal motor dysfunction in acute radiation enteritis. Am J Physiol Gastrointest Liver Physiol, 2007, 293: G121-127

4 Kountouras J, Zavos C. Recent advances in the management of radiation colitis. World J Gastroenterol, 2008, 14: 7289-7301

5 Phinney DG, Prockop DJ. Concise review: mesenchymal stem/multipotent stromal cells: the state of transdifferentiation and modes of tissue repair-current views. Stem Cells, 2007, 25: 2896-2902

6 Bernardo ME, Locatelli F, Fibbe WE. Mesenchymal stromal cells. Ann N Y Acad Sci, 2009, 1176: 101-117

7 Linard C, Ropenga A, Vozenin-Brotons MC, Chapel A, Mathe D. Abdominal irradiation increases inflammatory cytokine expression and activates NF-kappaB in rat ileal muscularis layer. Am J Physiol Gastrointest Liver Physiol, 2003, 285: 556-565

8 Herodin F, Roy L, Grenier N, Delaunay C, Bauge S, Vaurijoux A, Gregoire E, Martin C, Alonso A, Mayol JF, Drouet M. Antiapoptotic cytokines in combination with pegfilgrastim soon after irradiation mitigates myelosuppression in nonhuman primates exposed to high irradiation dose. Exp Hematol, 2007, 35: 1172-1181

9 Matsuu-Matsuyama M, Nakashima M, Shichijo K, Okaichi K, Nakayama T, Sekine I. Basic fibroblast growth factor suppresses radiation-induced apoptosis and TP53 pathway in rat small intestine. Radiat Res, 2010, 174: 52-61

10 Khizhniak SV, Prokhorova AA, Stepanova LI, Voitsitskii VM. Functioning of the antioxidant system in epithelial cells of small intestine under the influence of ionizing radiation of low dose rate. Radiats Biol Radioecol, 2011, 51: 684-688

11 Hauer-Jensen M, Wang J, Boerma M, Fu Q, Denham JW. Radiation damage to the gastrointestinal tract: mechanisms, diagnosis, and management. Curr Opin Support Palliat Care, 2007, 1: 23-29

12 Vyas D, Robertson CM, Stromberg PE, Martin JR, Dunne WM, Houchen CW, Barrett TA, Ayala A, Perl M, Buchman TG, Coopersmith CM. Epithelial apoptosis in mechanistically distinct methods of injury in the murine small intestine. Histol Histopathol, 2007, 22: 623-630

13 Freeman SL, MacNaughton WK. Nitric oxide inhibitable isoforms of adenylate cyclase mediate epithelial secretory dysfunction following exposure to ionising radiation. Gut, 2004, 53: 214-221

14 Merritt AJ, Allen TD, Potten CS, Hickman JA. Apoptosis in small intestinal epithelial from p53-null mice: evidence for a delayed, p53-independent G2/M-associated cell death after gamma-irradiation. Oncogene, 1997, 14: 2759-2766

15 Rotolo JA, Maj JG, Feldman R, Ren D, Haimovitz-Friedman A, Cordon-Cardo C, Cheng EH, Kolesnick R, Fuks Z. Bax and Bak do not exhibit functional redundancy in mediating radiation-induced endothelial apoptosis in the intestinal mucosa. Int J Radiat Oncol Biol Phys, 2008, 70: 804-815

16 Chang HJ, Maj JG, Paris F, Xing HR, Zhang J, Truman JP, Cardon-Cardo C, Haimovitz-Friedman A, Kolesnick R, Fuks Z. ATM regulates target switching to escalating doses of radiation in the intestines. Nat Med, 2005, 11: 484-490

17 Zhang L, Sun W, Wang J, Zhang M, Yang S, Tian Y, Vidyasagar S, Pena LA, Zhang K, Cao Y, Yin L, Wang W, Schaefer KL, Saubermann LJ, Swarts SG, Fenton BM, Keng PC, Okunieff P. Mitigation effect of an FGF-2 peptide on acute gastrointestinal syndrome after high-dose ionizing radiation. Int J Radiat Oncol Biol Phys, 2010, 77: 261-268

18 Shadad AK, Sullivan FJ, Martin JD, Egan LJ. Gastrointestinal radiation injury: symptoms, risk factors and mechanisms. World $\mathrm{J}$
Gastroenterol, 2013, 19: 185-198

19 Marshman E, Ottewell PD, Potten CS, Watson AJ. Caspase activation during spontaneous and radiation-induced apoptosis in the murine intestine. J Pathol, 2001, 195: 285-292

20 Sun Q, Ming L, Thomas SM, Wang Y, Chen ZG, Ferris RL, Grandis JR, Zhang L, Yu J. PUMA mediates EGFR tyrosine kinase inhibitor-induced apoptosis in head and neck cancer cells. Oncogene, 2009, 28: 2348-2357

21 Fei P, El-Deiry WS. P53 and radiation responses. Oncogene, 2003, 22: $5774-5783$

22 Qiu W, Leibowitz B, Zhang L, Yu J. Growth factors protect intestinal stem cells from radiation-induced apoptosis by suppressing PUMA through the PI3K/AKT/p53 axis. Oncogene, 2010, 29: 1622-1632

23 An MJ, Cheon JH, Kim SW, Park JJ, Moon CM, Han SY, Kim ES, Kim TI, Kim WH. Bovine colostrum inhibits nuclear factor kappaB-mediated proinflammatory cytokine expression in intestinal epithelial cells. Nutr Res, 2009, 29: 275-280

24 Lysy PA, Campard D, Smets F, Malaise J, Mourad M, Najimi M, Sokal EM. Persistence of a chimerical phenotype after hepatocyte differentiation of human bone marrow mesenchymal stem cells. Cell Prolif, 2008, 41: 36-58

25 Reger RL, Tucker AH, Wolfe MR. Differentiation and characterization of human MSCs. Methods Mol Biol, 2008, 449: 93-107

26 Tekkatte C, Vidyasekar P, Kapadia NK, Verma RS. Enhancement of adipogenic and osteogenic differentiation of human bone-marrowderived mesenchymal stem cells by supplementation with umbilical cord blood serum. Cell Tissue Res, 2012, 347: 383-395

27 Tao XR, Li WL, Su J, Jin CX, Wang XM, Li JX, Hu JK, Xiang ZH, Lau JT, Hu YP. Clonal mesenchymal stem cells derived from human bone marrow can differentiate into hepatocyte-like cells in injured livers of SCID mice. J Cell Biochem, 2009, 108: 693-704

28 Alexanian AR. An efficient method for generation of neural-like cells from adult human bone marrow-derived mesenchymal stem cells. Regen Med, 2010, 5: 891-900

29 Lin X, Peng P, Cheng L, Chen S, Li K, Li ZY, Mo YH, Zhou Z, Li M. A natural compound induced cardiogenic differentiation of endogenous MSCs for repair of infarcted heart. Differentiation, 2012, 83: $1-9$

30 Huang J, Zhang Z, Guo J, Ni A, Deb A, Zhang L, Mirotsou M, Pratt RE, Dzau VJ. Genetic modification of mesenchymal stem cells overexpressing CCR1 increases cell viability, migration, engraftment, and capillary density in the injured myocardium. Circ Res, 2010, 106: 1753-1762

31 Salem HK, Thiemermann C. Mesenchymal stromal cells: current understanding and clinical status. Stem Cells, 2010, 28: 585-596

32 Kudo K, Liu Y, Takahashi K, Tarusawa K, Osanai M, Hu DL, Kashiwakura I, Kijima $H$, Nakane A. Transplantation of mesenchymal stem cells to prevent radiation-induced intestinal injury in mice. J Radiat Res (Tokyo), 2010, 51: 73-79

33 Semont A, Francois S, Mouiseddine M, Francois A, Sache A, Frick J, Thierry D, Chapel A. Mesenchymal stem cells increase self-renewal of small intestinal epithelium and accelerate structural recovery after radiation injury. Adv Exp Med Biol, 2006, 585: 19-30

34 Yim YS, Noh YH, Kim DH, Lee MW, Cheuh HW, Lee SH, Yoo KH, Jung HL, Sung KW, Choi SJ, Oh WI, Yang YS, Koo HH. Correlation between the immature characteristics of umbilical cord blood-derived mesenchymal stem cells and engraftment of hematopoietic stem cells in NOD/SCID mice. Transplant Proc, 2010, 42: 2753-2758

35 Jin G, Prabhakaran MP, Ramakrishna S. Stem cell differentiation to epidermal lineages on electrospun nanofibrous substrates for skin tissue engineering. Acta Biomater, 2011, 7: 3113-3122

36 Mouiseddine M, Francois S, Semont A, Sache A, Allenet B, Mathieu N, Frick J, Thierry D, Chapel A. Human mesenchymal stem cells home specifically to radiation-injured tissues in a non-obese diabetes/severe combined immunodeficiency mouse model. $\mathrm{Br} \mathrm{J}$ Radiol, 2007, 80(Spec No 1): S49-55 
37 Quinones HI, List AF, Gerner EW. Selective exclusion by the polyamine transporter as a mechanism for differential radioprotection of amifostine derivatives. Clin Cancer Res, 2002, 8: 1295-1300

38 Bernardo ME, Emons JA, Karperien M, Nauta AJ, Willemze R, Roelofs H, Romeo S, Marchini A, Rappold GA, Vukicevic S, Locatelli F, Fibbe WE. Human mesenchymal stem cells derived from bone marrow display a better chondrogenic differentiation compared with other sources. Connect Tissue Res, 2007, 48: 132-140

39 Chen FH, Fu SY, Yang YC, Wang CC, Chiang CS, Hong JH. Combination of vessel-targeting agents and fractionated radiation therapy: the role of the SDF-1/CXCR4 pathway. Int J Radiat Oncol Biol Phys, 2013, 86: 777-784

40 Vagima Y, Lapid K, Kollet O, Goichberg P, Alon R, Lapidot T. Pathways implicated in stem cell migration: the SDF-1/CXCR4 axis. Methods Mol Biol, 2011, 750: 277-289

41 Hocking AM, Gibran NS. Mesenchymal stem cells: paracrine signaling and differentiation during cutaneous wound repair. Exp Cell Res, 2010, 316: 2213-2219

42 Zhang J, Gong JF, Zhang W, Zhu WM, Li JS. Effects of transplanted bone marrow mesenchymal stem cells on the irradiated intestine of mice. J Biomed Sci, 2008, 15: 585-594

43 Ben-Baruch A. Organ selectivity in metastasis: regulation by chemokines and their receptors. Clin Exp Metastasis, 2008, 25: 345-356

44 Sasaki M, Abe R, Fujita Y, Ando S, Inokuma D, Shimizu H. Mesenchymal stem cells are recruited into wounded skin and contribute to wound repair by transdifferentiation into multiple skin cell type. J Immunol, 2008, 180: 2581-2587

45 Lange C, Brunswig-Spickenheier B, Cappallo-Obermann H, Eggert K, Gehling UM, Rudolph C, Schlegelberger B, Cornils K, Zustin J, Spiess AN, Zander AR. Radiation rescue: mesenchymal stromal cells protect from lethal irradiation. PLoS One, 2011, 6: e14486

46 Mazhari R, Hare JM. Mechanisms of action of mesenchymal stem cells in cardiac repair: potential influences on the cardiac stem cell niche. Nat Clin Pract Cardiovasc Med, 2007, 4(Suppl 1): S21-26

47 Li Z, Jiang CM, An S, Cheng Q, Huang YF, Wang YT, Gou YC, Xiao L, Yu W J, Wang J. Immunomodulatory properties of dental tissue-derived mesenchymal stem cells. Oral Dis, 2014, 20: 25-34

48 Hoogduijn M J, Popp F, Verbeek R, Masoodi M, Nicolaou A, Baan C, Dahlke MH. The immunomodulatory properties of mesenchymal stem cells and their use for immunotherapy. Int Immunopharmacol, 2010, 10: 1496-1500

49 Marigo I, Dazzi F. The immunomodulatory properties of mesenchymal stem cells. Semin Immunopathol, 2011, 33: 593-602

50 Shi M, Liu ZW, Wang FS. Immunomodulatory properties and therapeutic application of mesenchymal stem cells. Clin Exp Immunol, 2011, 164: 1-8
51 Ge W, Jiang J, Arp J, Liu W, Garcia B, Wang H. Regulatory T-cell generation and kidney allograft tolerance induced by mesenchymal stem cells associated with indoleamine 2,3-dioxygenase expression. Transplantation, 2010, 90: 1312-1320

52 Bassi EJ, Aita CA, Camara NO. Immune regulatory properties of multipotent mesenchymal stromal cells: Where do we stand? World J Stem Cells, 2011, 3: 1-8

53 Najar M, Raicevic G, Fayyad-Kazan H, De Bruyn C, Bron D, Toungouz M, Lagneaux L. Immune-related antigens, surface molecules and regulatory factors in human-derived mesenchymal stromal cells: the expression and impact of inflammatory priming. Stem Cell Rev, 2012, 8: 1188-1198

54 Krampera M. Mesenchymal stromal cell 'licensing': a multistep process. Leukemia, 2011, 25: 1408-1414

55 Nemeth K, Leelahavanichkul A, Yuen PS, Mayer B, Parmelee A, Doi K, Robey PG, Leelahavanichkul K, Koller BH, Brown JM, Hu X, Jelinek I, Star RA, Mezey E. Bone marrow stromal cells attenuate sepsis via prostaglandin $\mathrm{E}(2)$-dependent reprogramming of host macrophages to increase their interleukin-10 production. Nat Med, 2009, 15: 42-49

56 Holley AK, Bakthavatchalu V, Velez-Roman JM, St Clair DK. Manganese superoxide dismutase: guardian of the powerhouse. Int J Mol Sci, 2011, 12: 7114-7162

57 Niu Y, Wang H, Wiktor-Brown D, Rugo R, Shen H, Huq MS, Engelward B, Epperly M, Greenberger JS. Irradiated esophageal cells are protected from radiation-induced recombination by MnSOD gene therapy. Radiat Res, 2010, 173: 453-461

58 Epperly MW, Wegner R, Kanai AJ, Kagan V, Greenberger EE, Nie S, Greenberger JS. Effects of MnSOD-plasmid liposome gene therapy on antioxidant levels in irradiated murine oral cavity orthotopic tumors. Radiat Res, 2007, 167: 289-297

59 Greenberger JS, Epperly MW. Antioxidant gene therapeutic approaches to normal tissue radioprotection and tumor radiosensitization. In Vivo, 2007, 21: 141-146

60 Epperly MW, Smith T, Zhang X, Goff JP, Franicola D, Greenberger B, Komanduri P, Wang H, Greenberger JS. Modulation of in utero total body irradiation induced newborn mouse growth retardation by maternal manganese superoxide dismutase-plasmid liposome (MnSOD-PL) gene therapy. Gene Ther, 2011, 18: 579-583

61 Ramanathan R, Misra UK. Radioprotection of lipogenesis from glucose (U-14C) and activities of pyridine nucleotide dehydrogenases in liver of gamma-irradiated female rats by cystamine. Int J Radiat Biol Relat Stud Phys Chem Med, 1975, 28: 35-43

62 Yang C, Chen HX, Zhou Y, Liu MX, Wang Y, Wang JX, Ren SP, Han Y, Wu BY. Manganese superoxide dismutase gene therapy protects against irradiation- induced intestinal injury. Curr Gene Ther, 2013, 13: 305-314

Open Access This article is distributed under the terms of the Creative Commons Attribution License which permits any use, distribution, and reproduction in any medium, provided the original author(s) and source are credited. 